\title{
A novel 3D-printed hybrid simulation model for robotic-assisted kidney transplantation (RAKT)
}

\author{
Raphael Uwechue ${ }^{1}$ (D) $\cdot$ Petrut Gogalniceanu ${ }^{1}$. Nicos Kessaris ${ }^{1} \cdot$ Nick Byrne $^{2,3} \cdot$ Pankaj Chandak $^{1,4}$. \\ Jonathon Olsburgh ${ }^{1,5} \cdot$ Kamran Ahmed $^{4,5} \cdot$ Nizam Mamode $^{1,4} \cdot$ Ioannis Loukopoulos $^{1}$
}

Received: 21 November 2017 / Accepted: 12 January 2018 / Published online: 27 January 2018

(c) The Author(s) 2018. This article is an open access publication

\begin{abstract}
Robotic-assisted kidney transplantation (RAKT) offers key benefits for patients that have been demonstrated in several studies. A barrier to the wider uptake of RAKT is surgical skill acquisition. This is exacerbated by the challenges of modern surgery with reduced surgical training time, patient safety concerns and financial pressures. Simulation is a well-established method of developing surgical skill in a safe and controlled environment away from the patient. We have developed a 3D printed simulation model for the key step of the kidney transplant operation which is the vascular anastomosis. The model is anatomically accurate, based on the CT scans of patients and it incorporates deceased donor vascular tissue. Crucially, it was developed to be used in the robotic operating theatre with the operating robot to enhance its fidelity. It is portable and relatively inexpensive when compared with other forms of simulation such as virtual reality or animal lab training. It thus has the potential of being more accessible as a training tool for the safe acquisition of RAKT specific skills. We demonstrate this model here.
\end{abstract}

Keywords Kidney transplantation $\cdot$ Simulation $\cdot$ Training $\cdot$ Robotic

Robotic-assisted kidney transplantation (RAKT) may provide enhanced patient recovery with comparable outcomes to standard open surgery. In addition, RAKT can increase access to kidney transplantation for specific end stage renal disease (ESRD) patients with morbid obesity [1]. The robotic platform provides high resolution three dimensional (3D) operative views enhancing stereotactic vision and improved depth perception [2], as well as superior

Raphael Uwechue

Raphael.uwechue@nhs.net

1 Department of Transplantation, Guy's Hospital, Renal Offices, 6th Floor Borough Wing, Great Maze Pond, London SE1 9RT, UK

2 Department of Medical Physics, Guy's and St Thomas' NHS Foundation Trust, London, UK

3 School of Biomedical Engineering and Imaging Sciences, King's College London, London, UK

4 MRC Centre for Transplantation, NIHR Biomedical Research Centre, King's Health Partners, King's College London, London, UK

5 Department of Urology, Guy's and St Thomas' NHS Foundation Trust, London, UK instrument handling with a vastly increased range of movement [3] compared to laparoscopic surgery. Consequently, RAKT is progressively expanding and showing promising results in centers of excellence $[4,5]$.

In robotic surgery, as in all other surgical approaches, patient safety is paramount. Skill acquisition for surgery in general, but particularly in robotic surgery, is hampered by time pressure [6], service provision and financial imperatives [7]. Simulation is a well-established method of teaching operative skills, reducing learning curves and providing transferable skills to the operating theatre [8] that improve operator performance [9]. Current simulation training is based on virtual reality simulators, in vivo animal models and cadaveric models [10]. These models remain costly and difficult to access. In addition, teams need to practice nontechnical skills including the robot setup, patient positioning and interacting dynamically with the robotic system to allow surgeons to operate successfully. These skills can be improved through simulation training [11].

3D-printing or rapid prototyping is a form of additive manufacturing technology that now has several recognized applications in the medical field including the creation of anatomical models for surgical planning, patient-specific 


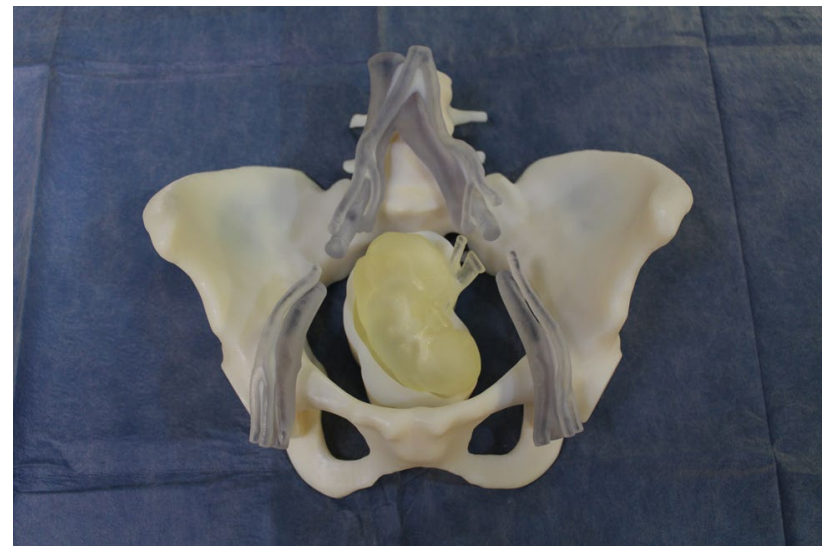

Fig. 1 Prosthetic cradles

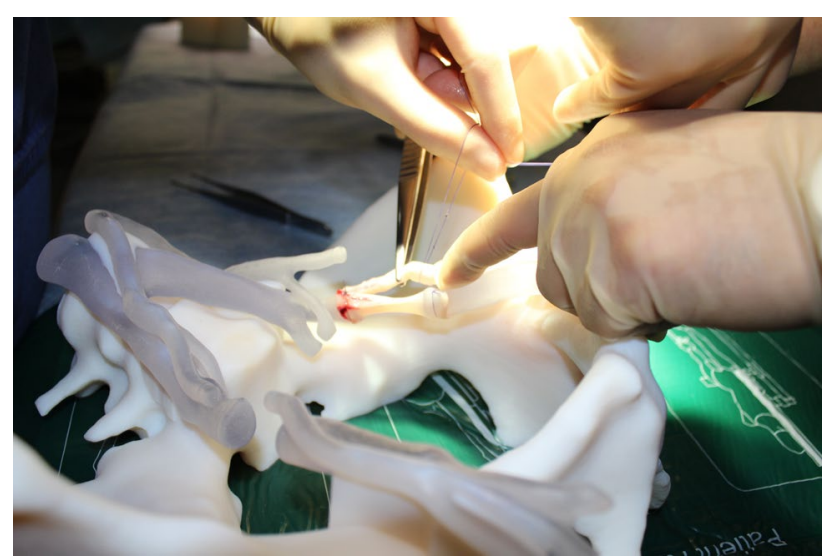

Fig. 2 Addition of deceased donor vessels to the cradles

surgical guides and custom prosthetic implants, as well as in medical education [12]. 3D printed models have now been demonstrated to show a high degree of anatomical accuracy, with implicit application as simulation training tools for the acquisition of technical skills [13] (Fig. 1).

A high-volume tertiary abdominal transplant unit in the UK planned to introduce a RAKT programme. To facilitate the new procedure, we created a hybrid simulation training model combining a 3D printed-moulage, integrated with deceased donor vessels. The purpose of the model was to optimise the surgeons training in vascular anastomoses in a timed and reproducible manner to allow objective assessment of their competencies (Fig. 2).

The key concept of this model is the use of human tissue to maximise the fidelity of the simulation exercise. The storage and use of deceased donor vessels for training purposes required institutional review and approval from the legally responsible organization under the United Kingdom Human Tissue Act 2008 legislation, which is NHS Blood and Transplant (NHSBT), was obtained. Eligible vessels for this tissue bank were iliac arteries and veins. They were collected when unused for either liver or pancreas transplants.

The prosthetic component consists of a synthetic 3D printed cradle of an anatomically accurate pelvis printed in life-size. In addition, 3D printing was used to create a kidney and a plinth to support the kidney in the correct operative position within the pelvis.

Recipient anatomy that was reproduced included:

- pelvis

- vertebral column from L4 to coccyx

- abdominal vasculature: aorta and IVC from L4 level,

- iliac vessels: the complete common iliac vessels, the internal iliac vessels terminating $3 \mathrm{~cm}$ distal from their origin; external iliac vessels to femoral bifurcation.

Donor anatomy included the body of the kidney and the renal artery and vein with an exposed length of $2 \mathrm{~cm}$.

Deceased donor iliac arteries and veins were obtained from the tissue bank. These were dissected and inter-positioned in the gap of the recipient's iliac artery and vein and secured with 2.0 Vicryl ligatures. Deceased donor iliac vessels were also mounted in a similar fashion on the 3D printed kidney's vascular stumps. The da Vinci Si robotic system was successfully docked in situ by the robotic team. Two robotic surgeons performed vascular anastomoses between the 'hybrid donor' renal vessels and the 'hybrid recipient's' iliac vessels using 6.0 prolene sutures. Mean anastomotic procedural time was 20 min per vessel. Vascular anastomotic patency was tested by intravascular injection of saline using a hypodermic needle. A good anastomotic patency and leak-resistance was demonstrated. Room and operating field video recording was undertaken for training feedback purposes. This facilitated group discussion, reflection and feedback.

The model offers several advantages. It is anatomically accurate offering geometrical fidelity and realistic spatial constraints. It utilizes deceased donor vessels to replicate reality. Deceased donor vessels also offer the advantage of being pre-screened for infection allowing it to be used in normal clinical operating theatres with the same operating robot used in clinical cases. In addition, the model can be used repeatedly, making it ideal for high-volume training, even in centers without 3D printing facilities. The use of video capture methods allows retrospective analysis of performance and structured debriefing for training, as well as revalidation purposes. Finally, the model can be deployed in situ to aid team-oriented training, as well as enhancing user immersion. Model development costs were acceptable, and the estimated reprint cost is around $£ 1000$. This represents a significant financial advantage compared with training on animal models or the cost of consumables in other forms of simulation (Fig. 3). 


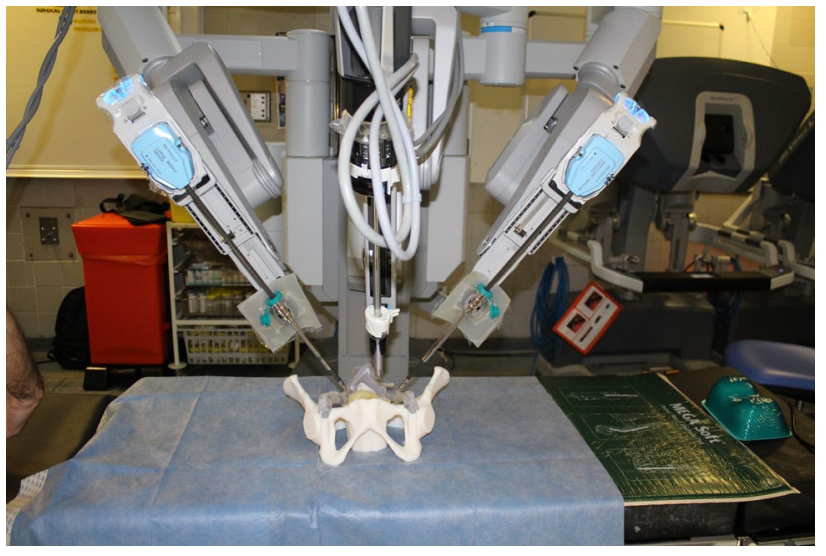

Fig. 3 Model in the operating theatre with the operating robot ready to use

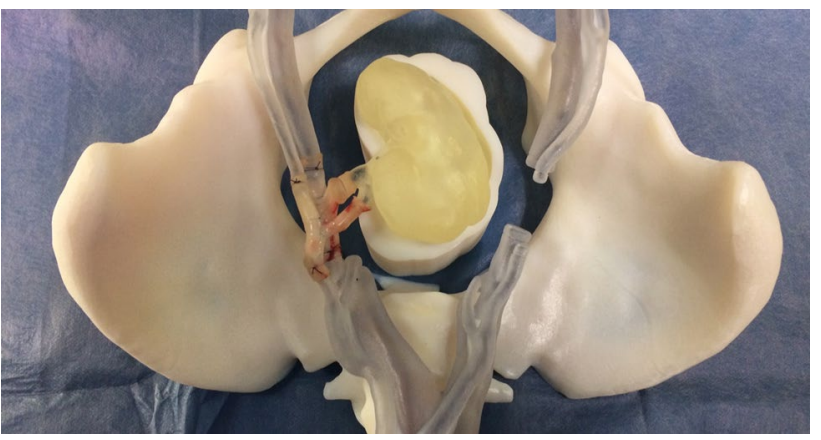

Fig. 4 Model with completed anastomoses

In the future, the model offers the opportunity to create bespoke patient-specific training, allowing surgeons to practice on clinically targeted operating fields. Fidelity may be further enhanced using the model in conjunction with an abdominal cavity simulator, which is already available. It has the potential to be incorporated into a future training curriculum for robotic transplant surgery (Fig. 4).

Main limitations of this concept include access to 3D printing facilities and deceased donor vessels. Another obstacle is that robotic theatre is usually available during non-office hours.

In summary, the model demonstrates how 3D-printedenhanced simulation can be used to bridge the gap between standard simulation and clinical practice in a simple and cost-effective manner. This represents a new step in hybrid RAKT simulation, combining novel 3D-printed technology with the time-tested benefits of using deceased donor tissue for high fidelity simulation training. The model is currently under validation by a multidisciplinary team of experts in robotic surgery.

\section{Compliance with ethical standards}

Funding This study has not received funding.

Conflict of interest Author RU declares that he has no conflict of interest. Author PG declares that he has no conflict of interest. Author NB declares that he has no conflict of interest. Author PC declares that he has no conflict of interest. Author JO declares that he has no conflict of interest. Author NK declares that he has no conflict of interest. Author KA declares that he has no conflict of interest. Author NM declares that he has no conflict of interest. Author IL declares that he has no conflict of interest.

Ethical approval All procedures performed in studies involving human participants were in accordance with the ethical standards of the institutional and/or national research committee and with the 1964 Helsinki declaration and its later amendments or comparable ethical standards.

Informed consent Informed consent was obtained from all individual participants included in the study.

Open Access This article is distributed under the terms of the Creative Commons Attribution 4.0 International License (http://creativeco mmons.org/licenses/by/4.0/), which permits unrestricted use, distribution, and reproduction in any medium, provided you give appropriate credit to the original author(s) and the source, provide a link to the Creative Commons license, and indicate if changes were made.

\section{References}

1. Oberholzer J, Giulianotti P, Danielson KK, Spaggiari M, BejaranoPineda L, Bianco F et al (2013) Minimally invasive robotic kidney transplantation for obese patients previously denied access to transplantation. Am J Transplant Off J Am Soc Transplant Am Soc Transpl Surgeons 13(3):721-728

2. Prasad SM, Prasad SM, Maniar HS, Chu C, Schuessler RB, Damiano RJ Jr (2004) Surgical robotics: impact of motion scaling on task performance. J Am Coll Surg 199(6):863-868

3. Kumar R, Hemal AK (2005) Emerging role of robotics in urology. J Minim Access Surg 1(4):202-210

4. Garcia-Roca R, Garcia-Aroz S, Tzvetanov I, Jeon H, Oberholzer J, Benedetti E (2017) Single center experience with robotic kidney transplantation for recipients with BMI of $40 \mathrm{~kg} / \mathrm{m}^{2}$ or greater: a comparison with the UNOS registry. Transplantation 101(1):191-196

5. Abaza R, Ghani KR, Sood A, Ahlawat R, Kumar RK, Jeong W et al (2014) Robotic kidney transplantation with intraoperative regional hypothermia. BJU Int 113(4):679-681

6. Datta ST, Davies SJ (2014) Training for the future NHS: training junior doctors in the United Kingdom within the 48-hour European working time directive. BMC Med Educ 14(Suppl 1):S12

7. Bridges M, Diamond DL (1999) The financial impact of teaching surgical residents in the operating room. Am J Surg 177(1):28-32

8. Larsen CR, Soerensen JL, Grantcharov TP, Dalsgaard T, Schouenborg L, Ottosen C et al (2009) Effect of virtual reality training on laparoscopic surgery: randomised controlled trial. BMJ (Clin Res Ed) 338:b1802

9. Torkington J, Smith SG, Rees BI, Darzi A (2001) Skill transfer from virtual reality to a real laparoscopic task. Surg Endosc 15(10):1076-1079

10. Schreuder HW, Wolswijk R, Zweemer RP, Schijven MP, Verheijen RH (2012) Training and learning robotic surgery, time for a more structured approach: a systematic review. BJOG 119(2):137-149 
11. Hull L, Sevdalis N (2015) Advances in teaching and assessing nontechnical skills. Surg Clin North Am 95(4):869-884

12. Tack P, Victor J, Gemmel P, Annemans L (2016) 3D-printing techniques in a medical setting: a systematic literature review. Biomed Eng Online 15(1):115
13. Pedersen TH, Gysin J, Wegmann A, Osswald M, Ott SR, Theiler L et al (2017) A randomised, controlled trial evaluating a low cost, 3D-printed bronchoscopy simulator. Anaesthesia 72(8):1005-1009 